
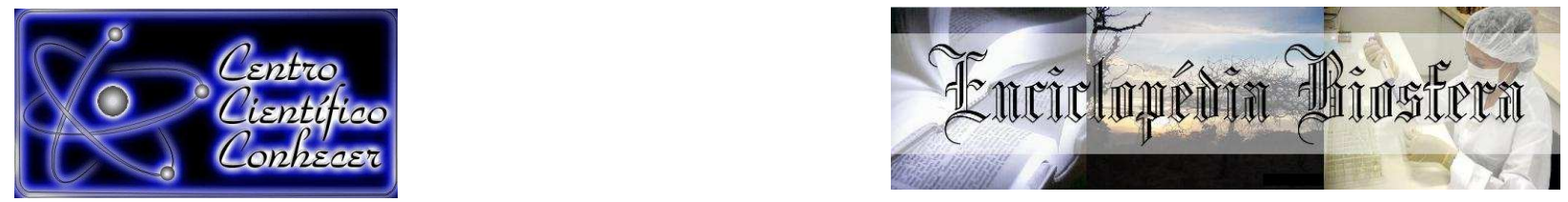

\title{
ATUAÇÃO FISIOTERAPÊUTICA NA DOR E QUALIDADE DE VIDA EM MULHERES COM DISFUNÇÃO TEMPOROMADIBULAR (DTM) DE ORIGEM MUSCULAR
}

\author{
João Paulo Leal ${ }^{1}$; Sonia Maria Marques Gomes Bertolini ${ }^{2}$ \\ ${ }^{1}$ Fisioterapeuta, graduado pelo Centro Universitário de Maringá- \\ UniCesumar/Maringá, Paraná - Brasil. \\ ${ }^{2}$ Doutora em Anatomia Humana e docente do Programa de Pós-Graduação Stricto \\ Senso em Promoção da Saúde (PPGPS), Centro Universitário de Maringá - \\ UniCesumar/Maringá, Paraná - Brasil. \\ sonia.bertolini@unicesumar.edu.br
}

Recebido em: 08/09/2015 - Aprovado em: 14/11/2015 - Publicado em: 01/12/2015 DOI: http://dx.doi.org/10.18677/Enciclopedia_Biosfera_2015_006

\begin{abstract}
A disfunção temporomandibular é uma doença multifatorial, com maior prevalência no sexo feminino. Além da dor, outros sinais e sintomas podem estar associados, como cansaço muscular, bloqueio, luxação além de ruídos do tipo estalos, estalidos e crepitação. Este estudo teve como objetivo investigar a redução da dor e melhora da qualidade de vida de pacientes com DTM, submetidos a laser e cinesioterapia. Foram incluídas mulheres com diagnóstico prévio de DTM muscular avaliadas em uma clínica de Odontologia e encaminhadas para tratamento fisioterapêutico à Clínica de Fisioterapia da UniCesumar. Para avaliação foi utilizada a Escala Visual Analógica (EVA) para graduação da dor, o questionário de qualidade de vida WHOQOL-BREF, o Índice Anamnésico de Fonseca e as provas de função dos músculos que movimentam a mandíbula. O tratamento constitui-se de um protocolo para a reabilitação, que foi aplicado rigorosamente por dez sessões, duas vezes por semana. Ao término do tratamento, as voluntárias foram reavaliadas e os resultados submetidos à análise estatística para comparação das variáveis pré e pósintervenção fisioterapêutica. Todas as pacientes apresentaram resultados satisfatórios em relação às variáveis testadas, embora sem significância estatística na comparação dos escores de qualidade de vida antes e após a intervenção. Conclui-se que os recursos fisioterapêuticos laser e cinesioterapia são eficazes no tratamento da disfunção Temporomandibular.
\end{abstract}

RESUMO

PALAVRAS - CHAVE: Disfunção da Articular, Fisioterapia, Qualidade de vida, Terapia por estimulação elétrica.

\section{PHYSIOTHERAPEUTIC PERFORMANCE IN PAIN AND WOMEN IN QUALITY OF LIFE WITH DYSFUNCTION OF ORIGIN temporomandibular MUSCLE}

\footnotetext{
ABSTRACT

Temporomandibular dysfunction is more prevalent in females multifactorial disease. Besides pain other signs and symptoms may be associated, such as muscle fatigue,
} 
blockage and dislocation as well as noises like pops, clicks and crackles. To investigate pain reduction and improved quality of life of patients with TMD underwent a protocol of physical therapy. Were included in this study women with a previous diagnosis of muscular TMD evaluated at the Clinic of Dentistry and sent to physical therapy for Physiotherapy Clinic of UniCesumar. The evaluation consisted of the application of the Visual Analogue Scale (VAS) to grade the pain, quality of life WHOQOL-BREF, Anamnesic Index of Fonseca and the evidence of function of the muscles that move the jaw. The treatment had a composition of several techniques establishing a protocol for rehabilitation, where the same protocol for ten sessions, twice a week has been rigorously conducted. After treatment, all patients were reevaluated and the results subjected to statistical analysis by the t-test for comparison of variables pre and post-intervention physical therapy. All patients had satisfactory results for the variables tested, although not statistically significant difference in scores of quality of life before and after the intervention.It is concluded that the therapy is of great importance in the treatment of temporomandibular dysfunction

KEYWORDS: Physical Therapy, Temporomandibular Joint Dysfunction, Quality of life, Electric stimulation therapy.

\section{INTRODUÇÃO}

A disfunção temporomandibular (DTM) é uma doença multifatorial, mais prevalente no sexo feminino (MARINHO et al., 2009). A dor é a principal queixa nos casos de DTM. Os pacientes podem apresentar cefaléia, dor à palpação da Articulação temporomandibular (ATM) e dos músculos da mastigação (MACHADO et al., 2010). Além da dor outros sintomas podem estar associados, como cansaço muscular, travamento e luxação da ATM, ruídos como estalos, estalidos e crepitação (MACHADO et al., 2010). Esses sintomas levam a uma piora na qualidade do sono desses pacientes (DRABOVICZ et al., 2012).

Como a musculatura cervical tem importante ligação com a ATM, ocorre frequente comprometimento muscular dessa região na DTM caracterizado pelo aumento da atividade muscular cervical mesmo em repouso, quando comparado a indivíduos sem a referida disfunção (MILANESI et al., 2011). Os pacientes com DTM também podem apresentar alterações posturais e biomecânicas (STRINI et al., 2009) e estudos indicam que indivíduos com DTM possuem maior número de desvios posturais quando comparados a indivíduos sem a disfunção (RIES \& BÉRZIN, 2008). Dentre os principais desvios encontram-se anteriorização da cabeça, aumento da lordose cervical e desnivelamento entre os ombros.

Outro fator determinante para o desenvolvimento ou agravamento da DTM são hábitos orais sendo os mais relatados ranger os dentes, roer as unhas, o costume de morder objetos como lápis, plásticos, palito de dente, ou qualquer outro objeto (BOVE et al., 2005). A qualidade de vida desses pacientes é afetada negativamente, devido à condição dolorosa, incapacidade de realizar determinadas funções, redução da vitalidade e do estado geral e mental de saúde, bem como alteração nos aspectos físicos, emocionais e sociais, todos ocasionados pela DTM, sendo a saúde mental influenciadora direta da redução da qualidade de vida, já que a ansiedade e a depressão são os principais fatores emocionais associados à DTM e a dor crônica (FERREIRA et al., 2009).

A avaliação e o tratamento devem ser feitos por uma equipe multiprofissional (MAFFEl et al., 2012) e conter uma anamnese específica e exame físico completo, com atenção às características físicas e emocionais para obtenção de um 
diagnóstico claro que possibilite o tratamento ideal (FERREIRA et al., 2009a). Exercícios terapêuticos e técnicas de correção postural têm sido efetivos na melhora do quadro clínico de pacientes com DTM associada a desvios posturais, promovendo redução da dor e da incapacidade funcional (BASSO et al., 2010). Esses exercícios trazem efeitos positivos a curto e longo prazo e devem estar associados a um programa de orientações domiciliares (MALUF et al. 2008), orientações essas que atuam preventivamente evitando afecções futuras, principalmente por abertura mandibular excessiva nos casos de hipermobilidade articular (PASINATO et al., 2011). A eletroestimulação também tem se mostrado efetiva na redução da dor em pacientes com DTM (GOMES et al., 2012). O laser de baixa frequência e a estimulação neural elétrica transcutânea (TENS) têm sido utilizados no tratamento da DTM alcançando melhora no quadro de dor dos pacientes (KATO et al., 2006).

Visto que há uma numerosa população com DTM muscular, acarretando em dor e redução da qualidade de vida, com consequências físicas, sócio-econômicas e psicológicas, são necessários estudos que investiguem a efetividade de intervenções fisioterapêuticas na promoção de analgesia e melhora da qualidade de vida. Pressupõe-se que a combinação de técnicas e recursos fisioterapêuticos podem promover analgesia e relaxamento muscular, melhorando consequentemente a qualidade de vida dos pacientes com DTM. Sendo assim, a presente pesquisa teve por objetivo investigar a redução da dor e a melhora da qualidade de vida de pacientes com DTM submetidos à laser e cinesioterapia.

\section{MATERIAL E METODOS}

O presente estudo é do tipo ensaio clínico, com 15 mulheres de idade entre 20 a 60 anos e diagnóstico clínico de DTM muscular, que procuraram a Clínica de Odontologia do UniCesumar e foram encaminhadas para o tratamento gratuito na Clínica de Fisioterapia da mesma instituição. A pesquisa foi realizada no período de março a junho de 2014.

Como critério de inclusão as pacientes deveriam apresentar diagnóstico clínico obtido por meio do RDC/TMD (Research Diagnostic Criteria for Temporomandibular Disorders) realizado pela Clínica de Odontologia do UniCesumar. Foram excluídas pacientes que não tinham o diagnóstico clínico de DTM muscular, que apresentaram alteração de sensibilidade, doenças neurológicas, fibromialgia, doenças cancerígenas e gestantes.

Após análise das fichas de avaliação as pacientes foram convidadas a participarem do projeto, sendo informadas quanto ao objetivo da pesquisa. As pacientes interessadas em participar da pesquisa passaram pela triagem quanto aos critérios de inclusão e exclusão do estudo. As selecionadas assinaram na Clínica de Fisioterapia um termo de consentimento livre e esclarecido (TCLE) antes da avaliação e do início do tratamento.

Para avaliação fisioterapêutica foi realizada a aplicação da Escala Visual Analógica (EVA) para graduação da dor da paciente (CIENA et al., 2008), o qual graduou o nível de dor antes e após cada sessão individualmente, assim como no início e fim do tratamento completo que incluiu 10 sessões. O questionário de qualidade de vida WHOQOL-BREF foi utilizado na comparação pré e póstratamento sendo classificados quanto aos domínios físicos, psicológicos, social e ambiental, sendo que em uma escala de 0 a 20, quanto maior o valor, melhor a qualidade de vida (FLECK et al., 2000). 
Para graduação quanto a severidade da DTM foi utilizado o Índice Anamnésico de Fonseca (PEDROSA, 2011), sendo classificado como sem disfunção (somatória de 0 a 15 pontos), DTM leve (somatória entre 20 a 40 pontos), DTM moderada (somatória entre 45 a 65 pontos) e DTM grave (somatória entre 70 a 100 pontos) analisada pré e pós-tratamento. Em relação à força muscular foi utilizado as provas de funções graduando a força dos músculos de 0 a 5 sendo, 0 sem contração muscular, 1-contração muscular palpável, 2- movimento articular com eliminação da gravidade, 3-movimento articular contra a gravidade, 4-movimento articular contra leve resistência e 5-força muscular normal contra resistência, analisados os grupos musculares de abertura, fechamento, protração, retrusão e lateralização direita e esquerda também foram aplicados pré e pós tratamento (KENDALL, 2007). Os demais dados foram extraídos do RDC de cada paciente.

Em relação ao tratamento, este teve duração de 10 sessões com frequência de duas vezes por semana e duração de 60 minutos e seguiu um protocolo préestabelecido durante todas as sessões conforme a sequência a seguir: I Alongamento de cervical e mobilização passiva mandibular; II - Desativação de ponto gatilho e liberação miofascial; III- Pompage cervical; IV- Laserterapia; VFortalecimento de muscular da região da ATM; VI - Eletroestimulação trascutânea em ATM e cervical;VII- Exercícios posturais em posição deitado, sentado e em pé.

Ao término desse programa todas as pacientes foram reavaliadas e as informações analisadas estatisticamente para comparação das variáveis pré e pós intervenção fisioterapêutica, sendo considerados significativos os resultados no valor de $p \leq 0,05$ através do programa Assistat 7.7, com a aplicação do teste t-Student.

\section{RESULTADOS}

Verificou-se que a maioria das mulheres $(60 \%)$ era casada, estudante $(34 \%)$, com média de idade de $32,3 \pm 10,49$ (Tabela 1 ).

TABELA 1. Características da amostra estudada.

\begin{tabular}{lcc}
\hline ESTADO CIVIL & $\mathrm{N}$ & $\%$ \\
\hline Solteira & 9 & $60 \%$ \\
Casada & 6 & $40 \%$ \\
TOTAL & 15 & $100 \%$ \\
\hline PROFISSÃO & QUANTIDADE & PORCENTAGEM(\%) \\
\hline Estudante & 5 & $34 \%$ \\
Advogada & 2 & $13,2 \%$ \\
Dona de casa & 2 & $13,2 \%$ \\
Vendedora & 2 & $13,2 \%$ \\
Aux. de escritório & 1 & $6,6 \%$ \\
Aux. Serigrafia & 1 & $6,6 \%$ \\
Professora & 1 & $6,6 \%$ \\
Recepcionista & 1 & $6,6 \%$ \\
TOTAL & 11 & $100 \%$ \\
\hline
\end{tabular}

Em relação à qualidade de vida verificou-se que houve melhora das pacientes, no entanto esta não foi estatisticamente significativa (Tabela 2). 
TABELA 2. Média, desvio padrão e valor de p, pré e pós-intervenção dos diferentes domínios do questionário de qualidade de vida.

\begin{tabular}{lccc}
\hline $\begin{array}{c}\text { QUESTIONÁRIO } \\
\text { WHOQUOL-BREF }\end{array}$ & \multicolumn{2}{c}{ Pré } & \multicolumn{2}{c}{ Pós } \\
Média & $\begin{array}{r}\text { Desvio } \\
\text { padrão }\end{array}$ & Média $\begin{array}{c}\text { Desvio } \\
\text { padrão }\end{array}$ & $\begin{array}{c}\text { Significativo } \\
\mathrm{p} \leq 0,05\end{array}$ \\
\hline Domínio físico (pt) & $12,06 \pm 2,45$ & $13,71 \pm 3,56$ & 0,15 \\
Domínio psicológico (pt) & $13,21 \pm 2,77$ & $13,87 \pm 2,46$ & 0,55 \\
Relação social (pt) & $14,43 \pm 3,25$ & $15,25 \pm 2,57$ & 0,53 \\
Meio Ambiente (pt) & $12,99 \pm 2,10$ & $13,94 \pm 2,38$ & 0,24 \\
\hline
\end{tabular}

Já quanto a função muscular verificou-se diferenças estatisticamente significantes pré e pós intervenção de todos os músculos testados (Tabela 3).

TABELA 3. Teste de função muscular pré e pós-intervenção em pacientes com DTM muscular.

\begin{tabular}{|c|c|c|c|c|c|}
\hline \multirow[b]{2}{*}{ MÚSCULOS } & \multicolumn{2}{|c|}{ Pré } & \multicolumn{2}{|c|}{ Pós } & \multirow{2}{*}{$\begin{array}{c}\text { Significativo } \\
p \leq 0,05\end{array}$} \\
\hline & Média & $\begin{array}{l}\text { Desvio } \\
\text { padrão }\end{array}$ & Média & $\begin{array}{l}\text { Desvio } \\
\text { padrão }\end{array}$ & \\
\hline Elevadores (pt) & \multicolumn{2}{|c|}{$4,53 \pm 0,63$} & \multicolumn{2}{|c|}{$4,93 \pm 0,25$} & $0,03^{*}$ \\
\hline Abaixadores (pt) & \multicolumn{2}{|c|}{$4,44 \pm 0,50$} & \multicolumn{2}{|c|}{$5 \pm 0$} & $<0,001^{*}$ \\
\hline Lateralizadores D. (pt) & \multicolumn{2}{|c|}{$3,86 \pm 0,83$} & \multicolumn{2}{|c|}{$4,86 \pm 0,35$} & $<0,001^{*}$ \\
\hline Lateralizadores E (pt) & \multicolumn{2}{|c|}{$3,93 \pm 0,79$} & \multicolumn{2}{|c|}{$4,86 \pm 0,35$} & $<0.001^{*}$ \\
\hline Protusores (pt) & \multicolumn{2}{|c|}{$4,26 \pm 0,59$} & \multicolumn{2}{|c|}{$4,86 \pm 0,35$} & $0.002^{*}$ \\
\hline Retrusores (pt) & \multicolumn{2}{|c|}{$4,00 \pm 0,84$} & \multicolumn{2}{|c|}{$4,86 \pm 0,35$} & $0,001^{*}$ \\
\hline
\end{tabular}

Verificou-se ainda efetividade da intervenção utilizada na variável dor e Índice de Fonseca. Com relação a este índice na pré-intervenção a DTM foi diagnosticada como grave $(74,6 \pm 14,69)$ e após a intervenção passou a ser classificada como leve $(40 \pm 12,53)$ considerando que a DTM com pontuação acima de 70 já é considerada grave e abaixo de 45 leve. Houve também diferença estatisticamente significante em relação a variável dor (Tabela 4).

TABELA 4. Média, desvio padrão e valor de $p$ das variáveis Índice de Fonseca e dor.

\begin{tabular}{lcccc}
\hline \multicolumn{1}{c}{ VARIÁVEIS } & \multicolumn{2}{c}{ Pré } & \multicolumn{2}{c}{ Pós } \\
Média & $\begin{array}{r}\text { Desvio } \\
\text { padrão }\end{array}$ & Média $\begin{array}{r}\text { Desvio } \\
\text { padrão }\end{array}$ & $\begin{array}{c}\text { Significativo } \\
\mathrm{p} \leq 0,05\end{array}$ \\
\hline Índice de Fonseca (pt) & $74,6 \pm 14,69$ & $40 \pm 12,53$ & $<0,001^{*}$ \\
EVA (pt) & $5,86 \pm 2,12$ & $0,78 \pm 1,61$ & $0,001^{*}$ \\
\hline
\end{tabular}




\section{DISCUSSÃO}

A Disfunção Temporomandibular é mais comum em mulheres com a escala de dor variando de leve a moderada o que acarreta uma série de fatores nocivos para a saúde (GERES et al., 2013). A prevalência de DTM em mulheres devido a relações fisiológicas, posturais e hormonais motivou a realização dessa pesquisa apenas com pacientes do sexo feminino.

O tratamento conservador proporcionado pela fisioterapia tem demonstrado grandes benefícios para diminuição das DTMs. Em um estudo de caso feito por ARENHART et al. (2013) verificou-se a eficiência da técnica de liberação de ponto gatilho, alongamento e fortalecimento muscular, atuando na fraqueza muscular e diminuindo a excessiva abertura bucal. Esse estudo assemelha-se ao realizado por CARVALHO et al. (2012), o qual era composto de 21 pacientes portadores da disfunção temporomandibular submetidos a exercícios cinesioterapêuticos. Os autores obtiveram resultados satisfatórios em relação a diminuição da dor, a melhora da biomecânica mastigatória e a qualidade de vida. A presente pesquisa embora com uma amostra menor, também evidenciou redução significativa na dor das pacientes.

BORIN et al. (2011), utilizando métodos fisioterapêuticos diferentes do proposto do presente estudo, observaram por meio do índice de Fonseca a diminuição da severidade da DTM em 40 pacientes. Vários são os resultados persuasivos na utilização da eletroterapia relacionada ao tratamento de DTM. O uso da estimulação neural elétrica transcutânea (TENS) mostra-se eficaz em quadros álgicos quando aplicado corretamente pelo terapeuta (GROSMANN et al. 2012), fato igualmente constatado no estudo DE CARVALHO et al. (2012). O presente estudo mostrou que ambas as técnicas utilizadas na mesma sessão produziram efeito analgésico imediato.

Os exercícios contra resistidos para fortalecimento muscular aplicado na fase aguda da DTM têm como objetivo o deslocamento e o reposicionamento do disco articular na ATM. Em uma DTM muscular há uma fraqueza da musculatura que envolve a articulação proporcionando uma facilitação no deslocamento do disco. Em uma segunda fase do tratamento o fortalecimento muscular proporciona ganho de estabilidade do disco articular dentro da fossa articular na ATM consequentemente há uma diminuição do atrito entre disco e côndilo mandibular que causam quadros dolorosos (FRICTION \& DUBNER 2003). O ganho de força muscular de todos os grupos analisados na pesquisa pós-intervenção fisioterapêutica demonstra a eficácia do fortalecimento na biomecânica levando ao quadro de diminuição ou ausência de dor.

Os resultados encontrados no presente estudo demostram que todas as pacientes obtiveram a melhora na qualidade de vida segundo questionário WHOQOL-BREF, porém as diferenças nos escores obtidos antes e após o tratamento não foram significantes em nenhum dos domínios.

Em relação aos exercícios posturais, estes foram escolhidos pelo fato dos pacientes com DTM apresentarem tensão muscular na região cervical proporcionando compensações que alteram toda biomecânica correta da mastigação (FERREIRA et al., 2009b). A estabilidade postural corrige o centro de pressão postural (CCP) devolvendo a homeostase articular temporomandibular (NEIVA et al., 2012). Mesmo não analisando diretamente a postura, foi possível perceber que os exercícios posturais impactaram positivamente na dor das pacientes. 
Ao analisar a escala visual analógica pré e pós-tratamento de cada sessão foi possível identificar que após a $5^{\circ}$ sessão já havia melhora sensível da dor, porém, vale ressaltar que caso o tratamento fosse interrompido antes da $10^{\circ}$ sessão possivelmente o quadro de DTM retornaria. Sendo assim, mostra-se de suma importância assiduidade e o comprometimento do paciente na conclusão do tratamento proposto.

\section{CONCLUSÃO}

Conclui-se que os recursos fisioterapêuticos laser e cinesioterapia são eficazes no tratamento da disfunção Temporomandibular e sugere-se estudos que também utilizem a variável postural como forma de quantificar os resultados da intervenção fisioterapêutica.

\section{REFERÊNCIAS}

ARENHART, R.; LAZAROTTO, R.; THOMÉ, K. Tratamento fisioterapêutico na disfunção temporomandibular: um estudo de caso.Revista Fisisenectus, v.1, n.1, p. 109-117, 2013.

BASSO, D.; CORRÊA, E.; SILVA, A.M. Efeito da reeducação postural global no alinhamento corporal e nas condições clínicas de indivíduos com disfunção temporomandibular associada a desvios posturais. Revista Fisioterapia e Pesquisa, v.17, n.1, p.63-68, jan/mar. 2010.

BORIN, G.S; CORRÊA, E.C.; SILVA, A.M.T.; MILANESI, J.M. Acupuntura como recurso terapêutico na dor e na gravidade da desordem Temporomandibular. Revista Fisioterapia e Pesquisa, v.18, n.3, p. 217-22 ,jul/set. 2011.

BOVE, S.R.K.; GUIMARÃES, A.S.; SMITH, R.L. Caracterização dos pacientes de um ambulatório de disfunção temporomandibular e dor orofacial. Revista Latinoamericano de Enfermagem; v.13, n.5, p.686-691, Set/Out. 2005.

CARVALHO, A.G.C.; ANDRIOLA, A.E.M.; NASCIMENTO, J.A.; OLIVEIRA, E.A.; CARDIA, M.C.G.; LUCENA N.M.G. Influência da cinesioterapia e da estimulação elétrica nervosa trascutãnea (TENS) em portadores de disfunção temporomandibular. Revista Brasileira de Ciência e Saúde.v.16, n.2, p.17-24, 2012.

CIENA A.P.; GATTO R.; PACINI V.C.; PICANÇO V.V.; MAGNO I.M.N.; LOTH E.A. Influência da intensidade da dor sobre as respostas nas escalas unidimensionais de mensuração da dor em uma população de idosos e de adultos jovens. Semina : Ciências Biológicas e da Saúde, v. 29, n.2, p.201-212, jul./dez. 2008.

DRABOVICZ, P.V.S.M.; SALLES, V.; PAULO E.M.; DRABOVICZ, P.E.M.; FONTES, M.J.F. Assessmentofsleepquality adolescentswithtemporomandibulardisorders.Jornal de Pediatria, v.88, n.2, p.169172, 2012.

FERREIRA, F.V.; FERREIRA, F.V.; PERONI, A.B.F.; TABARELLI, Z. Desordens tempomandibulares: uma abordagem fisioterapêutica e odontológica. Revista Stomatos, v.15,n.28, p. 27-37, 2009a. 
FERREIRA, K.D.M.; GUIMARÃES, J.P.; BATISTA, C.H.T.; FERRAZ JÚNIOR, A.M.L.; FERREIRA, L.A. Fatores psicológicos relacionados à sintomatologia crônica das desordens temporomandibulares - revisão de literatura. Revista Faculdade de Odontologia, v. 14, n. 3, p. 262-267, set/dez. 2009b.

FLECK, M.P.A.; LOUZADA, M.X.; CHACHAMOVICH, E.; VIEIRA, L.S.; PINZON, V. Aplicação da versão em português do instrumento abreviado de avaliação da qualidade de vida "WHOQOL-bref". Revista Saúde Pública, v.34, n.2, p.177-183, 2000.

FRICTION,J.R.; DUBNER,R. Dor orofacial e desordens temporomandibulares. 1 ed.São Paulo: Santos,. p. 335- 336, 2003.

GERES, G.S.; PACHION, C.A.S.; MASSELI, M.R.; FERREIRA, D.M.A.; GOMES, D.C.A.; PACHION, F.S.M.; KOIKE, T.E. Análise de condições clínicas em estudantes com disfunção temporomandibular. Revista Terapia manual, v.11, n.53, p. 361-366, 2013.

GOMES, N.C.M.C.; BERNI-SCHWARZENBECK, K.C.S.; PACKER, A. C.RODRIGUES-BIGATON, D. Efeito da estimulação elétrica de alta voltagem catódica sobre a dor em mulheres com DTM. Revista Brasileira de Fisioterapia, v.16, n.1, p.10-15, jan/fev. 2012.

GROSSMANN, E.; TAMBARA, J.S.; GROSSMANN, T.K.; SIQUEIRA, J.T.T. O uso da eletroestimulação nervosa trascutãnea na disfunção temporamandibular: artigo de revisão. Revista Dor, v.13, n.3, p. 271-276, 2012.

KATO, M.T.; KOGAWA, E.M.; SANTOS, C.N.; RODRIGUES, P.C. Tens and lowlevel laser therapy in the management of temporomandibular disorders.Journal of Applied Oral Science, v.14, n.2, p.130-135, 2006.

KENDALL, F.P; MCCREARY, E.K.; PROVANCE, P.G. Músculos: provas e funções com postura e dor. 5. ed.São Paulo: Manole, p.116, 2007.

MACHADO, I.M.; PIALARISSI, P.R.; MINICI, T.D.; ROTONDI, J.; FERREIRA, L.P. Relação dos Sintomas Otológicos nas Disfunções Temporomandibulares. Arquivos Internacionais de Otorrinolaringologia,v.14, n.3, p.274-279, Jul/Ago/Set. 2010.

MAFFEI, C.; MELLO, M.M.; BIASE, N.G.; PASETTI, L.; CAMARGO, P.A.; SILVÉRIO, K.C.A.; GONÇALVES, M.I.R. Videofluoroscopic evaluation of mastication and swallowing in individuals with TMD. Brazilian Journal of Otorhinolaryngol, v.78, n.4, p.24-8, 2012.

MALUF, S.A.; MORENO, B.G.D.; ALFREDO, P.P.; MARQUES, A.P.; RODRIGUES, G. Exercícios terapêuticos nas desordens temporomandibulares: uma revisão de literatura. Revista Fisioterapia e Pesquisa, v.15, n.4, p.408-415, out./dez. 2008 
MARINHO, C.C.; CRUZ, F.L.G.; LEITE,F.P.P. Correlação entre a oclusão e a disfunção Temporomandibular. Revista Odonto Ciência, v. 17, n. 34, p.49-55, jul./dez. 2009.

MILANESI, J.M.; CORRÊA, E.C.R.; BORIN, G.S.; SOUZA, J.A.; PASINATO, F. Atividade elétrica dos músculos cervicais e amplitude de movimento da coluna cervical em indivíduos com e sem DTM.Revista Fisioterapia e Pesquisa, v.18, n.4, p. 317-322, out/dez. 2011.

NEIVA, N.B.; VILELLA, O.V.; SILVA, G.C.H.; AMARAL, A.D. Posture alterations related to temporomandibular joint dysfunction. Journal of Dentistry and Oral Hygiene, v.4, n.1, p.1-5, 2012.

PASINATO, F.; SOUZA, J.A.; CORRÊA, E.C.R.; SILVA, A.M.T.Disfunçãotêmporomandibular e hipermobilidade articular generalizada: aplicação de critérios diagnósticos. Brazil Journal Otorhinolaryngology, v.77, n.4, p. 418-425, abr/jun. 2011.

PEDROSA, A.S. Propriedades de medida do índice anamnésico de fonseca. 2011. 39f. Dissertação (Mestrado em fisioterapia). Universidade Cidade de São Paulo, São Paulo, 2011.

RIES, L.G.K.; BÉRZIN, F. Analysis of the postural stability in individuals with or ithout signs and symptoms of temporomandibular disorder.Brazilian Oral Research, v.22, n.4, p.378-383, 2008.

STRINI, P.J.S.A.; MACHADO, N.A.G.; GORRERI, M.C.; FERREIRA, A.F.; SOUSE, G.C.; FERNADES NETO, A.J. Postural evaluation of patients with temporomandibular disorders under use of occlusal splints. Journal of Applied Oral Science, v.17, n.5, p.539-543, 2009. 